\title{
Adaptive Parametrization of Multivariate B-splines for Image Registration
}

\author{
Hansen, Michael Sass; Glocker, Benjamin; Navab, Nassir; Larsen, Rasmus
}

Published in:

Proceedings of CVPR

Link to article, DOI:

10.1109/CVPR.2008.4587760

Publication date:

2008

Document Version

Publisher's PDF, also known as Version of record

Link back to DTU Orbit

Citation (APA):

Hansen, M. S., Glocker, B., Navab, N., \& Larsen, R. (2008). Adaptive Parametrization of Multivariate B-splines for Image Registration. In Proceedings of CVPR (pp. 1-8). IEEE. https://doi.org/10.1109/CVPR.2008.4587760

\section{General rights}

Copyright and moral rights for the publications made accessible in the public portal are retained by the authors and/or other copyright owners and it is a condition of accessing publications that users recognise and abide by the legal requirements associated with these rights.

- Users may download and print one copy of any publication from the public portal for the purpose of private study or research.

- You may not further distribute the material or use it for any profit-making activity or commercial gain

- You may freely distribute the URL identifying the publication in the public portal

If you believe that this document breaches copyright please contact us providing details, and we will remove access to the work immediately and investigate your claim 


\section{Adaptive Parametrization of Multivariate B-splines for Image Registration}

\author{
Michael Sass Hansen and \\ Rasmus Larsen \\ Technical University of Denmark \\ Lyngby, Denmark \\ msh@imm.dtu.dk
}

\begin{abstract}
We present an adaptive parametrization scheme for $d y$ namic mesh refinement in the application of parametric image registration. The scheme is based on a refinement measure ensuring that the control points give an efficient representation of the warp fields, in terms of minimizing the registration cost function. In the current work we introduce multivariate B-splines as a novel alternative to the widely used tensor B-splines enabling us to make efficient use of the derived measure.

The multivariate B-splines of order $n$ are $C^{n-1}$ smooth and are based on Delaunay configurations of arbitrary $2 D$ or $3 D$ control point sets. Efficient algorithms for finding the configurations are presented, and B-splines are through their flexibility shown to feature several advantages over the tensor B-splines. In spite of efforts to make the tensor product B-splines more flexible, the knots are still bound to reside on a regular grid. In contrast, by efficient nonconstrained placement of the knots, the multivariate Bsplines are shown to give a good representation of inhomogeneous objects in natural settings.

The wide applicability of the method is illustrated through its application on medical data and for optical flow estimation.
\end{abstract}

\section{Introduction}

Image alignment is a challenging task due the inferior number of constraints compared to the number of unknowns. One approach to overcome this problem is to reduce dimensionality of the problem. Hence, parametric image registration has become quite popular and has been successfully applied to many applications. The two main advantages are (i) the reduced number of parameters which makes the problem tractable from an optimization point of view and (ii) often the transformation model inherently provides some smoothness properties on the warping field. In order to be able to capture the present deformations, hierarchical approaches have been considered where the number

\author{
Ben Glocker and \\ Nassir Navab \\ Technische Universität München \\ München, Germany
}

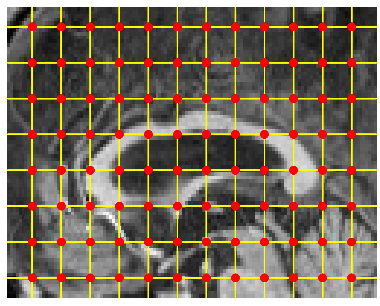

(a) Regular B-spline grid

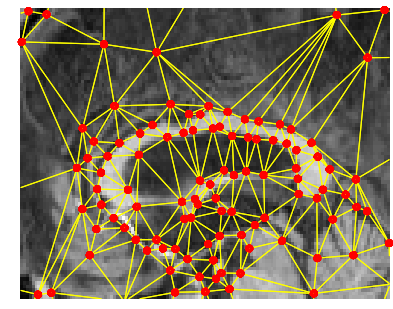

(b) Multivariate B-spline knots
Figure 1. In (b) it is seen how the multivariate B-splines can adopt to the structure in the image.

of parameters is successively increased during the optimization process. However, these update schemes often follow some heuristic approaches, for instance in mesh-based models by simply reducing the spacing between control points and thus do not incorporate any quantitative or qualitative measurement about the state of the registration. One can claim, that the refinement strategy has a significant influence on the solution and should be guided by some knowledge obtained directly from the images and/or the optimization process. Furthermore, one can claim that the transformation model should be flexible enough and dynamically adjustable in order to reflect such extracted knowledge during image alignment. Therefore, we propose a framework for parametric image registration which allows us on one hand to assess the quality of the current solution locally and on the other hand we can estimate the potential improvement by a local refinement of the parameter set. Since, one could expect that such an improvement will spatially vary over the image domain, there is a need for flexible transformation models.

B-splines are popular in numerous applications because of achievable smoothness properties and the local support. Tensor product B-splines were introduced for modeling free form deformations (FFD) in the context of computer graphics [13]. The method was improved by using hierarchical B-splines $[5,16]$. The same subdivision scheme was used in medical image registration [11]. However, the tensor prod- 
uct B-splines are defined uniformly on a grid, and in the search for a more local control of the representation detail, the notion of an active set of knots was introduced in [12]. Still, such an simulation approach consists of locally uniform grids which cannot represent the imaged objects in an appropriate way.

Cootes et al. created a minimum description length optimization scheme for the placement of control points of triangular piecewise affine interpolation basis functions [3]. However, the method is defined as a groupwise method, and it relies heavily on the calculation of the inverse deformation. Recently Chandrashekara et al. proposed a parameterization based on a subdivision scheme, also to obtain a limited number of parameters [10].

We instead propose to use the multivariate B-splines as presented by Neamtu, to obtain a flexible and smooth warp interpolation function with local support[7]. They are capable of representing fields more densely in some areas, while keeping the number of degrees of freedoms small for an efficient optimiation.

Dahmen et al. [4] introduced the so called DMS-splines or triangular B-splines based on the multivariate simplex splines and auxiliary knots. Franssen et al. [6] described a new method for efficient calculation of triangular B-splines using an evaluation graph. Recently, Wang et al. [15] introduced DMS-splines in computer vision for nonrigid registration with rigid parts that defined by manual landmarks. The most recent multivariate B-splines were introduced by Neamtu [7], and they rely heavily on the new concept of Delaunay configurations [8].

The remainder of this paper is organized as follows: first we will present the general framework for parametric image registration. Based on this, we derive our adaptive parametrization scheme. In Section 4 we present the concept behind multivariate B-splines and their use in our framework for image registration. Experimental results are shown in Section 5 while the last Section concludes our paper.

\section{Parametric Image Registration}

The image registration problem can be formulated as the minimization of the functional $\mathcal{J}$ given by

$$
\mathcal{J}[I, R, \varphi]=\mathcal{D}[I, R ; \varphi]+\mathcal{S}[\varphi]
$$

where $I$ is an $s$-dimensional deformable image, $R$ is the target/reference image, $\varphi$ is the mapping from $R$ into corresponding points in $I$. In the present work we are focusing on parametric image registration, which means that the warps can formally be represented by

$$
\varphi: \mathcal{R}^{M} \rightarrow\left(f: \mathcal{R}^{s} \rightarrow \mathcal{R}^{s}\right)
$$

which means that the parameters of $\varphi$ determines what the warp will look like. We will think of $\varphi$ as being represented as

$$
\varphi=\sum_{i} \boldsymbol{c}_{i} K_{i}(\boldsymbol{x})
$$

where $K_{i}$ are the basis functions, and the vectors $\boldsymbol{c}_{i}=$ $\left[c_{i 1} \ldots c_{i s}\right]^{T}$ are the parameters associated with the basis function.

$\mathcal{D}[I, R ; \varphi]$ is a similarity measure and $\mathcal{S}[\varphi]$ is a regularization measure, and we will discuss these functionals in the context of parametric warp representation, in the following sections.

\subsection{Similarity Measures}

As a similarity measure the sum of squared distances (SSD) is used, but this could be exchanged with any other common similarity measures.

$$
\mathcal{D}[I, R ; \varphi]=\int_{\Omega}[I \circ \varphi(x)-R(x)]^{2} d x
$$

For the subsequent analysis we shall need some of the derivatives of $\mathcal{D}[I, R ; \varphi]$. The Gâteaux derivative of $\mathcal{D}$ is given by

$$
d_{\boldsymbol{\varphi}, \boldsymbol{v}} \mathcal{D}[I, R ; \boldsymbol{\varphi}]=\int_{\Omega}\langle\boldsymbol{f}(\boldsymbol{x}, \boldsymbol{\varphi}), \boldsymbol{v}\rangle_{\mathcal{R}^{s}} d \boldsymbol{x}
$$

where $f$ can be perceived as registration forces in the image, and is given by

$$
\boldsymbol{f}(\boldsymbol{x}, \boldsymbol{\varphi}(\boldsymbol{x}))=(I \circ \boldsymbol{\varphi}(\boldsymbol{x})-R(\boldsymbol{x})) \cdot \nabla I \circ \boldsymbol{\varphi}(\boldsymbol{x}),
$$

which is also denoted as the driving force of the registration process.

The variation $\boldsymbol{v}(\boldsymbol{x})$ is restricted to the subspace spanned by the basis functions. The derivative with respect to a warp parameter $c_{i j}$ is given by

$$
\frac{\partial \mathcal{D}}{\partial c_{i j}}=d_{\boldsymbol{\varphi}, \frac{\partial \boldsymbol{\varphi}}{\partial c_{i j}}} \mathcal{D}[I, R ; \boldsymbol{\varphi}]=\int_{\Omega}\left\langle\boldsymbol{f}(\boldsymbol{x}, \boldsymbol{\varphi}), \frac{\partial \boldsymbol{\varphi}}{\partial c_{i j}}\right\rangle_{\mathcal{R}^{s}} d \boldsymbol{x}
$$

\subsection{Regularization}

Often some prior knowledge is available about the presented image registration problem. This prior knowledge can generally be expressed as a differential regularizer $\mathcal{B}$, and some Sobolev norm.

$$
\mathcal{S}[\boldsymbol{\varphi}]=\int_{\Omega}\langle\mathcal{B}[\boldsymbol{u}], \mathcal{B}[\boldsymbol{u}]\rangle_{\mathcal{R}^{s}} d \boldsymbol{x},
$$

where $\varphi=I d+\boldsymbol{u}$. This norm has a Gâteaux derivative given by

$$
d_{u ; v} \mathcal{S}[\boldsymbol{u}]=\int_{\Omega}\langle\mathcal{A}[\boldsymbol{u}](\boldsymbol{x}), \boldsymbol{v}(\boldsymbol{x})\rangle_{\mathcal{R}^{d}} d \boldsymbol{x},
$$


where $\mathcal{A}=\mathcal{B}^{*} \mathcal{B}$. For the present work the commonly used elastic regularizer is chosen, which can be represented by

$$
\mathcal{B}[\boldsymbol{u}]=\left[\begin{array}{cc}
\sqrt{\mu} & 0 \\
0 & \sqrt{2 \mu+\lambda}
\end{array}\right]\left[\begin{array}{c}
\nabla \times \boldsymbol{u} \\
\nabla \cdot \boldsymbol{u}
\end{array}\right]
$$

from which $\mathcal{A}=\mu \Delta \boldsymbol{u}+(\lambda+\mu) \nabla(\nabla \cdot \boldsymbol{u})$. We can again form the derivative with respect to the parameter

$$
\frac{\partial \mathcal{S}[\boldsymbol{u}]}{\partial c_{i j}}=\int_{\Omega}\left\langle\mathcal{A}[\boldsymbol{u}], \frac{\partial \boldsymbol{u}}{\partial c_{i j}}\right\rangle d \boldsymbol{x}
$$

\section{Adaptive Parametrization}

After minimizing (7) the following equation must hold for the reached optimum.

$$
\frac{\partial \mathcal{J}[I, R ; \boldsymbol{\varphi}]}{\partial c_{i j}}=\frac{\partial \mathcal{D}[I, R ; \boldsymbol{\varphi}]}{\partial c_{i j}}+\frac{\partial \mathcal{S}[\boldsymbol{u}]}{\partial c_{i j}}=0 .
$$

This is the parameterized version of the variational optimum

$$
\boldsymbol{f}(\boldsymbol{x})+\mathcal{A}[\boldsymbol{u}]=0, \boldsymbol{x} \in \Omega
$$

Now observe that (11) can be interpreted as an averaged projection of the variational optimum (12)

$\frac{\partial \mathcal{D}}{\partial c_{i j}}+\frac{\partial \mathcal{S}[\boldsymbol{u}]}{\partial c_{i j}}=\int_{\Omega}\left\langle f(\boldsymbol{x}, \varphi)+\mathcal{A}[\boldsymbol{u}], \frac{\partial \boldsymbol{u}}{\partial c_{i j}}\right\rangle d \boldsymbol{x}=0$

where the projection is performed on to the support of the warp parameter. This projection, derived from (3), is given by

$$
\frac{\partial \boldsymbol{u}}{\partial c_{i j}}(\boldsymbol{x})=\boldsymbol{e}_{j} K_{i}(\boldsymbol{x}), \quad \boldsymbol{x} \in \Omega
$$

where $\boldsymbol{e}_{j}$ denotes the unit vector along the $j$ th dimension and $K_{i}$ is the basis function associated with the parameter $c_{i j}$. In popular terms, each of our parameters is designed to achieve the variational optimum (12) in average only, and for this reason it seems obvious to pose the question; how well is the variational optimum achieved? We propose to measure this fitness of a basis function in terms of the improvement we could achieve by replacing the basis function with several more local basis functions. We will assume that the basis function can be refined into several similar basis functions, only with a smaller support, and the response of these local basis functions can be modelled by applying a Gaussian filter on the force residues $\left\langle f(\boldsymbol{x}, \varphi)+\mathcal{A}[\boldsymbol{u}], \frac{\partial \boldsymbol{u}}{\partial c_{i j}}\right\rangle$. The filter response will model the changes that can be achieved with a basis function refinement, when we choose the kernel size to be close to that of the refined basis functions.

Let $\mathbf{1}=\boldsymbol{e}_{1}+\ldots+\boldsymbol{e}_{s}$ then the above consideration lead us to define a refinement measure $F$ by

$$
F\left[B_{I}\right]=\int_{\Omega} \mathcal{F}_{\sigma_{I}} *\left(\left\langle f(\boldsymbol{x}, \varphi)+\mathcal{A}[\boldsymbol{u}], \mathbf{1} B_{I}(\boldsymbol{x})\right\rangle\right)^{2} d \boldsymbol{x},
$$

where $\mathcal{F}_{\sigma_{I}}$ * denotes convolution with a Gaussian of a kernel width $\sigma_{I}$ which should be chosen in the order of $\sigma_{I}=$ $\left[\operatorname{vol}\left[B_{I}\right]\right]^{1 / s} / 4$, where $\operatorname{vol}\left[B_{I}\right]$ is the volume of the convex hull of the basis function support. A perfect fit would mean that the only way (12) was not satisfied would be noise, and $F\left[B_{I}\right]$ would then be 0 . However, if there is spatial coherency in the forces, $f(\boldsymbol{x}, \varphi)+\mathcal{A}[\boldsymbol{u}]$, then $F\left[B_{I}\right]$ will give an output suggesting to do a refinement of the mesh. This criteria should guide the refinement.

In order to make efficient use of the proposed adaptation scheme, we need a set of basis functions with spatially varying local support.

\section{Multivariate B-splines}

The multivariate B-splines presented in this paper are using a basis of simplex splines. These splines are smooth functions with local support. Several ways exist for composing sets of simplex splines to form a partition of unity, the most recent one, and the one presented in the current work, being multivariate B-splines based on Delaunay configurations [7]. Simplex splines and Delaunay configurations are briefly discussed here. Throughout the dimension is still denoted by $s$ and the degree of the splines by $n$.

\subsection{Simplex Splines}

Simplex splines are defined iteratively with the zeroth order spline defined on the simplex of $s+1$ knots, e.g. a triangle in $2 \mathrm{D}$.

$$
\begin{array}{rl} 
& M\left(\boldsymbol{x} \mid\left\{\boldsymbol{x}_{1}, \ldots, \boldsymbol{x}_{s+1}\right\}\right)= \\
1 / \operatorname{vol}\left[\left\{\boldsymbol{x}_{1}, \ldots, \boldsymbol{x}_{s+1}\right\}\right], & \boldsymbol{x} \in \operatorname{int}\left[\left\{\boldsymbol{x}_{1}, \ldots, \boldsymbol{x}_{s+1}\right]\right. \\
0 & \boldsymbol{x} \notin \operatorname{int}\left[\left\{\boldsymbol{x}_{1}, \ldots, \boldsymbol{x}_{s+1}\right]\right.
\end{array}
$$

where $M$ denotes the spline value, $\boldsymbol{x}$ is a point we wish to evaluate, $\boldsymbol{x}_{i}$ are the knot points of the simplex spline, int refers to the convex hull of the set of points, and vol is the volume of the convex hull.

The recurrence relation of the higher order simplex splines is given by

$$
\begin{array}{r}
M(\boldsymbol{x} \mid \boldsymbol{X})=\sum_{\boldsymbol{x}_{i} \in \boldsymbol{X}} \lambda_{i} M\left(\boldsymbol{x} \mid \boldsymbol{X} \backslash\left\{x_{i}\right\}\right), \text { where } \\
\sum_{\boldsymbol{x}_{i} \in \boldsymbol{X}} \boldsymbol{\lambda}_{i}=1, \quad \sum_{\boldsymbol{x}_{i} \in \boldsymbol{X}} \lambda_{i} \boldsymbol{x}_{i}=\boldsymbol{x} .
\end{array}
$$

Here $X$ is a set of $n+s+1$ knots (corresponding to a simplex spline $M$ of order $n$, and $\boldsymbol{\lambda}$ is seen to be the barycentric coordinates of the points.

The simplex splines are $n-1$ smooth on the convex hull of the knots, when none three of the simplex splines are collinear [7]. Examples of 2D simplex splines of different orders are illustrated in Figure 2. 


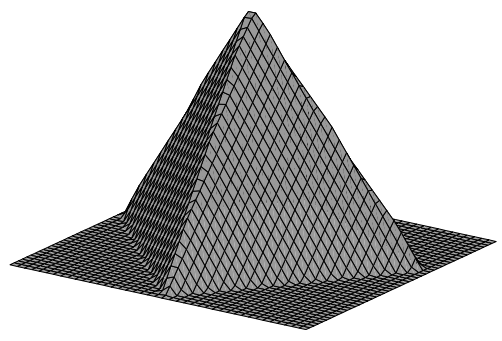

(a) Linear simplex spline

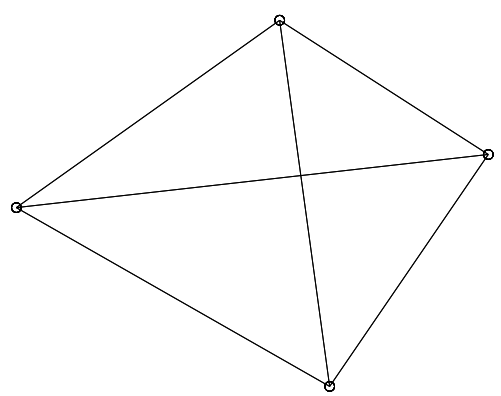

(d) 4 knots for linear simplex

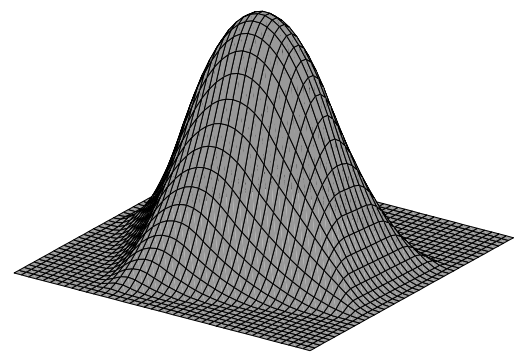

(b) Quadratic simplex spline

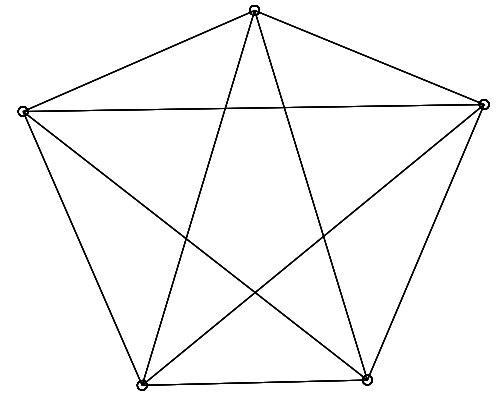

(e) 5 knots for quadratic simplex

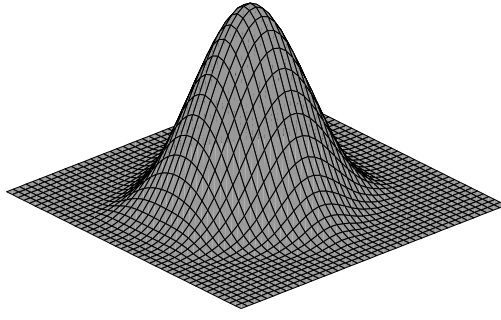

(c) Cubic simplex spline

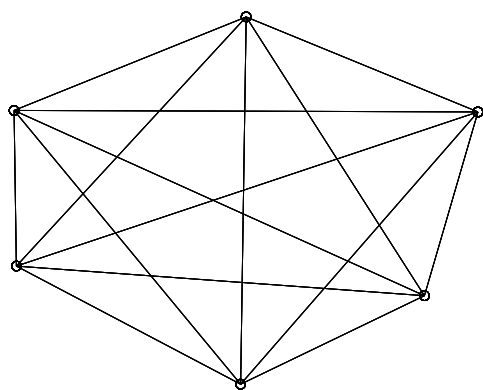

(f) 6 knots for cubic simplex

Figure 2. Simplex splines of increasing order and smoothness.

For the elastic regularization discussed in Section B the directional derivatives are needed, and the two first directional derivatives of the simplex splines are

$$
\begin{array}{r}
d_{\boldsymbol{v}} M(\boldsymbol{x} \mid X)=n \sum_{i=0}^{s} \mu_{i}(\boldsymbol{v}) M\left(\boldsymbol{x} \mid X \backslash\left\{\boldsymbol{x}_{i}\right\}\right) \\
d_{\boldsymbol{v}_{2}} d_{\boldsymbol{v}_{1}} M(\boldsymbol{x} \mid X)=n \sum_{i=0}^{s} \mu_{i}\left(\boldsymbol{v}_{2}\right) d_{\boldsymbol{x}, \boldsymbol{v}_{1}} M\left(\boldsymbol{x} \mid X \backslash\left\{\boldsymbol{x}_{i}\right\}\right), \\
\text { where } \sum_{i=0}^{s} \mu_{i}=0, \quad \sum_{i=0}^{s} \mu_{i} \boldsymbol{x}_{i}=x
\end{array}
$$

Complexity The complexity of the multivariate B-splines can be expressed in terms of the number of $M_{0}$ nodes visited, and this is $n^{s+1}$ if a naive implementation is chosen. However, through fingerprinting visited nodes, this graph can be reduced considerably [6]. When calculating the interpolation values, the derivatives can be calculated simultaneously.

\subsection{Delaunay Configurations}

For choosing appropriate sets of knots for simplex splines, the Delaunay configuration is needed. The Delaunay configuration is a generalization of the Delaunay triangulation, where the circumscribed sphere contains exactly $n$ points. Denote a given Delaunay configuration of $n$th order by $\Delta_{n}$. Let the set of all interior point sets, with $n$ points in each be denoted by $\mathcal{I}$. Then a set of interior points $I \in \mathcal{I}$ is associated with a set of boundary point sets $\mathcal{B}(I)=\left\{B \mid(B, I) \in \Delta_{n}\right\}$. We now define the multivariate B-spline associated with $n$ interior points $I$ as [7]

$$
B_{I}=\sum_{B \in \mathcal{B}(I)} \operatorname{vol}[B] M(. \mid B \cup I)
$$

This normalization ensures a partition of unity, i.e.

$$
\sum_{I \in \mathcal{I}} B_{I}(x)=1, \quad x \in \mathcal{R}^{s}
$$

Using these multivariate B-splines as a basis for describing the deformation field, the field can be defined as

$$
\boldsymbol{\varphi}(\boldsymbol{x})=\boldsymbol{x}+\sum_{I \in \mathcal{I}} \boldsymbol{c}_{I} B_{I}(\boldsymbol{x}), \quad \boldsymbol{c}_{I}, \boldsymbol{x} \in \mathcal{R}^{s}
$$

Silveira et al. have shown a strategy for efficient computation of the Delaunay configurations [14].

\subsection{Mesh Layout and Refinement}

For deriving an initial guess for placement of the knots, it can be noted that (13) seems most likely to yield big values, in areas where the forces $\boldsymbol{f}(\boldsymbol{x}, \boldsymbol{\varphi})$ attain big values. In terms, we expect the most changes to our deformation field to happen where the gradient is bigger, since the force and the gradient are proportional, (6). For the initial coarse distribution of knots, we propose to distribute them randomly, with a prior density based on the image gradient of the reference. Additionally, we add knots sequentially, according to the following update scheme 


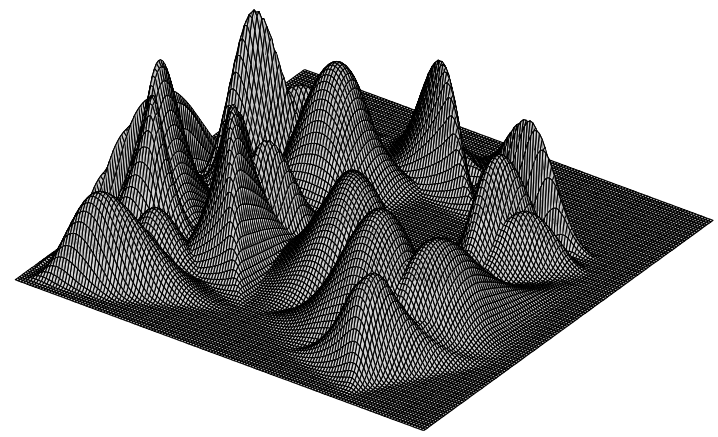

(a) Multivariate B-spline

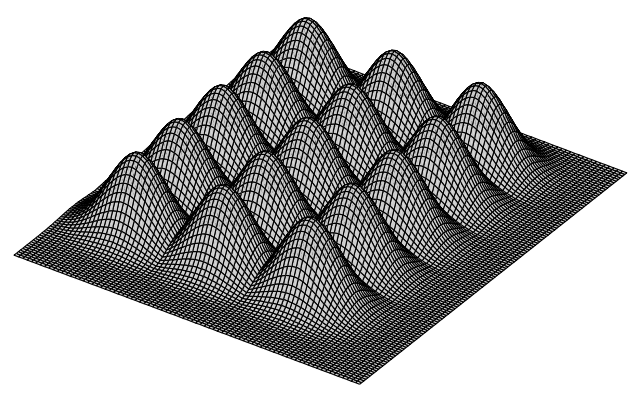

(b) Tensor product B-spline

Figure 3. Illustration of the flexible kernel of Multivariate Bsplines compared to the tensor product B-spline.

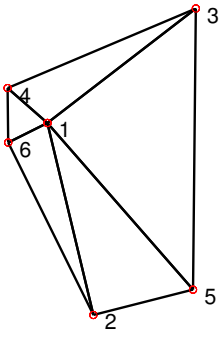

(a) A cell

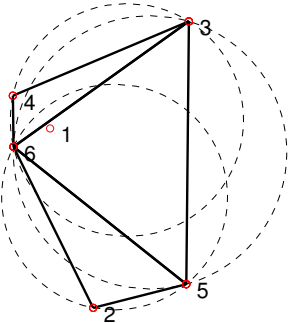

(b) The Delaunay configurations

Figure 4. Illustration of a cell of a first order Delaunay configuration. Notice how point $\mathbf{1}$ is inside the 3 triangles circumscribed spheres forming the Delaunay triangulation of its connected points. They constitute the whole cell with $\mathbf{1}$ as an inside point.

$$
\begin{aligned}
& f\left(\boldsymbol{x}_{j+1} \mid R,\left\{\boldsymbol{x}_{1}, \ldots, \boldsymbol{x}_{j}\right\}\right) \approx f\left(\boldsymbol{x}_{j+1} \mid R\right) \prod_{i=1}^{j} f\left(\boldsymbol{x}_{j+1} \mid \boldsymbol{x}_{i}\right) \\
\propto & \frac{(\nabla R)^{T} \nabla R}{\|\nabla R\|^{2}} \prod_{i=1}^{j}\left[1-\exp \left\{-\frac{\left(\boldsymbol{x}-\boldsymbol{x}_{i}\right)^{T}\left(\boldsymbol{x}-\boldsymbol{x}_{i}\right)}{2 \sigma}\right\}\right],
\end{aligned}
$$

where $\nabla R$ is the gradient of the reference, and $\sigma$ is a kernel diameter, where the kernel in effect limits the chance of a second point being place in the immediate vicinity of a knot. This function can be perceived as a prior probability for placing knots, and they can be placed either according to the maximum, or according to a random sampling. This did not seem to have big effect on results though, due to the subsequent mesh refinement.

We propose to make the mesh refinement based on subdivision of the grid of the knots, where the B-splines are expected to give most improvement in the cost function, when subdivided, according to the refinement measure derived in Section 3. To increase the resolution around a given B-spline, we subdivide the inner points and their triangulated neighbors. To enforce better subdivision, the subdivided knots are tracked to a nearby gradient, using a localized version of $f\left(\boldsymbol{x}_{j+1} \mid R,\left\{\boldsymbol{x}_{1}, \ldots, \boldsymbol{x}_{j}\right\}\right)$, localized by a Gaussian. The process of subdivision can be performed repeatedly until a sufficiently good resolution is obtained.

The local forces, as well as the effect of smoothing is illustrated in Figure 5. In Figure 5 (b), the forces are seen to be directed towards the corpus callosum both upwards and downwards. In average they even out, so there is no net force on the parameter. In Figure 5(a) the differences are seen to be intact after the smoothing. Therefore the refinement function has an output, and the B-spline is selected for subdivision.

\section{Evaluation and Results}

To support the methodological considerations presented in the previous section, we applied our implemented method on two data sets, both quite different in nature. Our image registration algorithm is implemented for $2 \mathrm{D}$ images, due to the 2D nature of our applications, but all observations and equations stated in the current work are equally valid for three dimensional data.

\subsection{Groupwise Corpus Callosa Registration}

To demonstrate the presented method, we did a groupwise registration of 62 mid-sagittal cross-section MR images of the corpus callosum brain structure. To apply the refinement measure (13) to a group of images, instead of on a single image, we calculate the sum over the whole data set for each B-spline, in order to ensure that our mesh refinement yields the biggest groupwise improvement in terms of minimization of the sum of squared differences.

This data set is part of the LADIS (Leukoaraiosis and DISability) study [9], a pan-European study involving 12 hospitals and more than 700 patients. Each corpus callosum has been manually segmented by a clinical expert. We used these segmentations for further assessment of the method (see Table 1). 


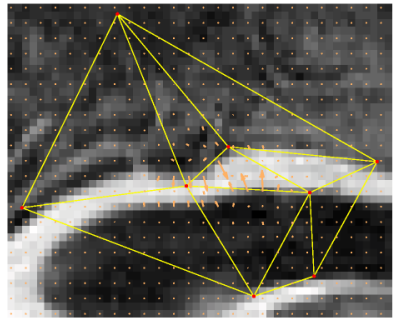

(a) Local 'forces' acting on parameter

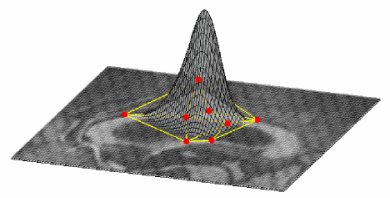

(c) The B-spline basis function

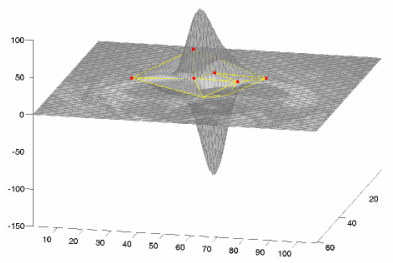

(e) Smoothed projection of forces

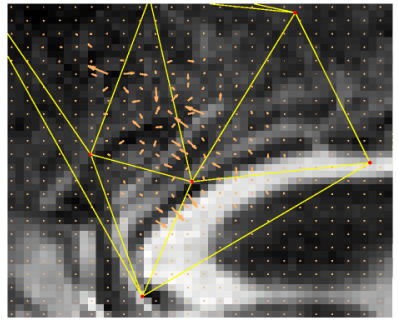

(b) Local 'forces' acting on parameter

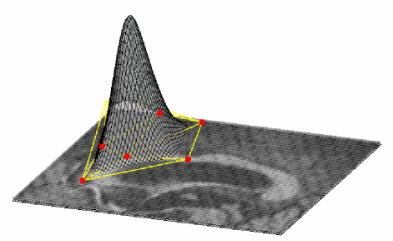

(d) The B-spline basis function

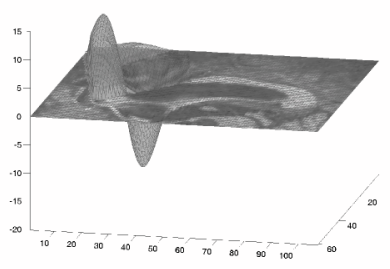

(f) Smoothed projection of forces
Figure 5. Illustration of a basis function that will be updated (left column), and one where the impact is not big enough (right column). Notice how (e) and (f) differ by an order of magnitude.

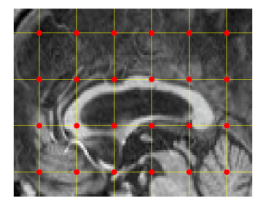

(a) Initial placement

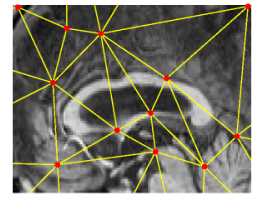

(d) Initial placement

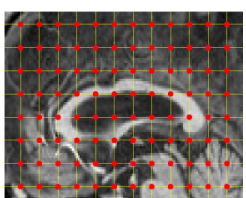

(b) 2nd level

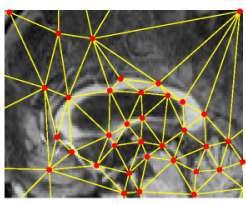

(e) 2nd level

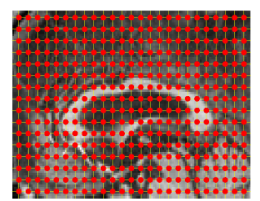

(c) 3rd level

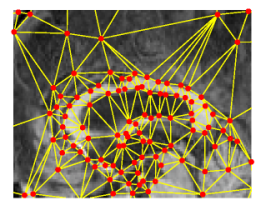

(f) 3rd level
Figure 6. The grid refinement for a uniform setting and our nonuniform setting on the corpus callosum data is illustrated here. Our refinement is based on (13). Notice how the structures are nearly segmented by the knots in the non-uniform case.

All images are registered to one image in the group, and in Figure 7 some results of the image registration algorithm are seen. The results of our implementation are compared to the results obtained by rigid alignment and by using an FFD algorithm, which is based on tensor product B-splines. Our

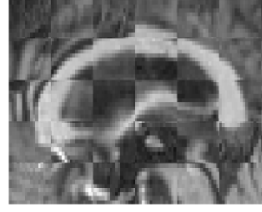

(a) Rigid

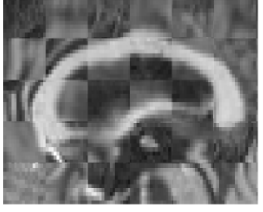

(b) FFD

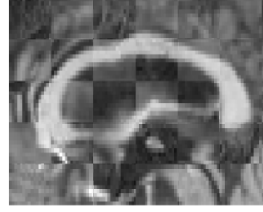

(c) Multivariate
Figure 7. Checkerboard illustrations of registration results, where the images are tiled from reference and registered image.

\begin{tabular}{|c|c|c|c|c|c|c|}
\hline Method & $\begin{array}{c}\text { DICE } \\
\text { median }\end{array}$ & Sens. & Spec. & N & $\begin{array}{c}\text { Init. } \\
\text { time }\end{array}$ & $\begin{array}{c}\text { Run } \\
\text { time }\end{array}$ \\
\hline Multi. & 0.85 & 0.91 & 0.99 & 407 & 32 & 149 \\
FFD & 0.83 & 0.91 & 0.98 & 640 & 1 & 221 \\
Rigid & 0.70 & 0.80 & 0.98 & & & \\
\hline
\end{tabular}

Table 1. Comparison of the warped segmentations and the reference segmentation after registration using a rigid transformation, an FFD based method, and our multivariate method. Notice how the initialization time (seconds) is larger, but the group-wise running time is smaller for the presented method, due to the reduced number of nodes.

quantitative studies showed that the FFD with slightly more control points gave inferior results, but still a significant improvement from the results of the rigid registration alone. The pre-computations of the multivariate splines were a lot more time consuming, but in the group-wise registration, this time was regained in the optimization step, due to the reduced number of control points.

\subsection{Optical Flow Estimation}

Baker et al. recently presented a database for comparison of optical flow results, where the quantitative results of optical flow estimations can be compared to other available algorithms [2]. The optical flow problem resembles the image registration problem a lot, in the sense that we seek to identify correspondences, or flow, across image pairs.

To test the current method on a different application we did the optical flow estimation on the three data sets, from the set, where ground truth flow is available, namely the image pairs named Dimetrodon, Venus and Yosemite. In Figure 8 the images are shown with the final grid resolution and the estimated flow field is shown along with the ground truth flow fields.

The results are summarized in Table 2. It is seen that our implementation performs significantly better on the one data set than any of the other methods in question, and slightly worse than the best, for the two other data sets.

\section{Conclusion}

The current work has several contributions to the field of parametric image registration. 


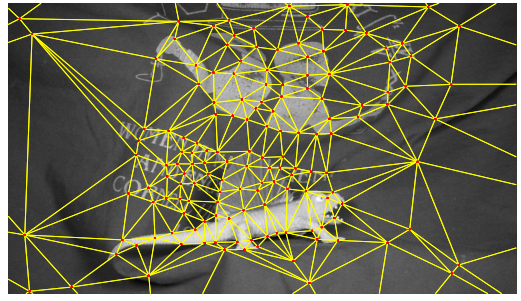

(a) Dimetrodon image with knot mesh

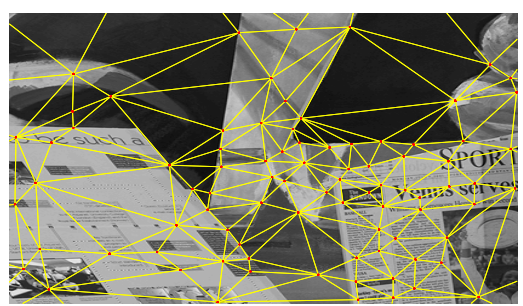

(d) Venus image with knot mesh

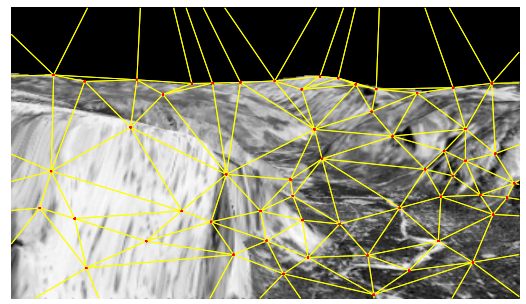

(g) Yosemite with knot mesh

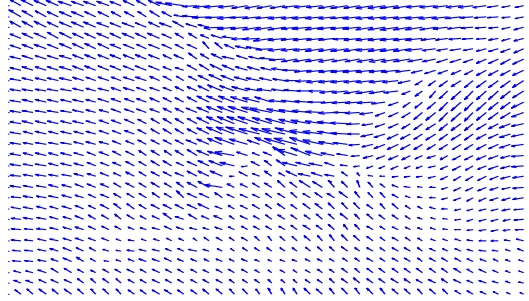

(b) Estimated Dimetrodon flow field

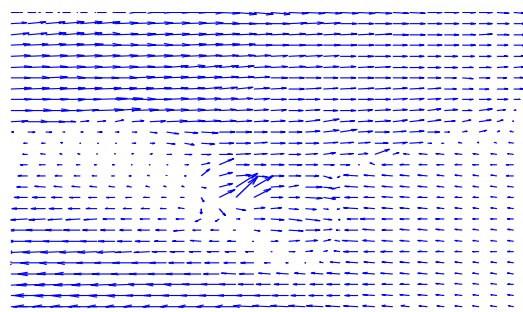

(e) Estimated Venus flow field

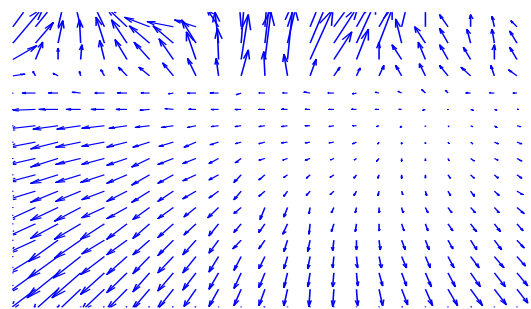

(h) Estimated Yosemite flow field

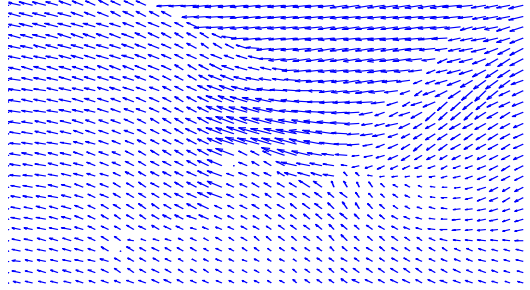

(c) True Dimetrodon flow field

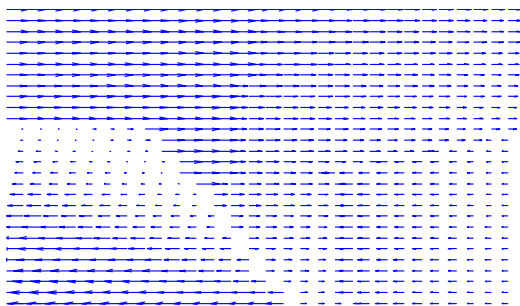

(f) True Venus flow field

Figure 8. One image of the optical data sets, and the estimated and true flow fields are shown.

\begin{tabular}{|c||cc|cc|cc|}
\hline \multicolumn{1}{|c||}{ Method } & \multicolumn{2}{|c|}{ Dimetrodon } & \multicolumn{2}{c|}{ Venus } & \multicolumn{2}{c|}{ Yosemite } \\
& Av. End point & Avg. Angular & Agv. End point & Avg. Angular & Avg. End point & Avg. Angular \\
\hline Current Method & 0.20 & 4.09 & 0.72 & 10.74 & 0.16 & 3.10 \\
\hline Bruhn et al. & 0.43 & 10.99 & 0.51 & 8.73 & 0.08 & 1.69 \\
Black and Anandan & 0.35 & 9.26 & 0.55 & 7.64 & 0.15 & 2.65 \\
Pyramid LK & 0.37 & 10.27 & 1.03 & 14.61 & 0.20 & 5.22 \\
Zitnick et al. & 0.94 & 15.82 & 0.85 & 15.48 & 0.68 & 11.09 \\
Mediaplayer & 0.55 & 30.10 & 1.08 & 11.42 & 0.47 & 18.50 \\
\hline
\end{tabular}

Table 2. Optical flow evaluation results compared to other contemporary methods

With an offset in variational optimization theory, we have derived the parametric version of the elastic potential regularization and in effect illustrating how the whole class of differential operator derived regularizers, i.e. curvature and bending energy, can be easily implemented in a parametric setting.

With the same methodology we have analyzed the inherent smoothing or averaging cost, of selecting warp parameterizations at a specific kernel resolution, in comparison to choosing a finer resolution of the warp kernels. Based on these observations we have proposed a refinement measure, which is shown to be efficient for guiding the local mesh layout.

Though both previous results are useful in their own merits, we have introduced the recently emerged multivariate B-splines based on Delaunay configurations, to the field of image registration. With the combination of our refinement measure and the local flexibility of the multivariate B-splines, we are able to automatically refine the warp field in areas where it results in the minimization of the registration cost function. In effect we get something close to a segmentation of objects allowing for better local control, even where very inhomogeneous areas share a border.

\section{A. Implementation}

The inverse compositional optimization approach by Baker et al. was used in our implementation to achieve a fast optimization [1]. We obtain a minimum by iteratively 
minimizing

$$
\begin{aligned}
\mathcal{J}_{i c}(\boldsymbol{c})= & \sum_{\boldsymbol{x}}\left(R(\boldsymbol{\varphi}(\boldsymbol{x}, \Delta \boldsymbol{c}))-I(\boldsymbol{\varphi}(\boldsymbol{x}, \boldsymbol{c}))^{2}\right. \\
& +\sum_{i} F_{i}^{2}\left(\boldsymbol{c t}-\frac{\partial \boldsymbol{c}^{\prime}}{\partial \Delta \boldsymbol{c}} \Delta \boldsymbol{c}\right)
\end{aligned}
$$

with respect to $\Delta c$, and the regularizer is expressed as $F_{i}^{2}(\boldsymbol{c})$ as derived in Appendix B. The parameter updating of $c$ is done according to

$$
\varphi\left(x, c^{\prime}\right) \leftarrow \varphi(x, c) \circ \varphi^{-1}(x, \Delta c) .
$$

\section{B. Elastic regularization on Multivariate B- splines}

In this section the elastic regularizer and the Lamé operator are derived for the multivariate B-splines, as. To use the inverse compositional algorithm for the image registration, we formulate the regularizer as a sum of functions on the parameters

$$
S[\boldsymbol{u}]=\int_{\Omega} \frac{\lambda}{2}(\nabla \cdot \boldsymbol{u})^{2}+\frac{\mu}{4} \sum_{i, j=1}^{s}\left[\frac{\partial \boldsymbol{u}_{i}}{\partial \boldsymbol{x}_{j}}+\frac{\partial \boldsymbol{u}_{j}}{\partial \boldsymbol{x}_{i}}\right]^{2} d x
$$

Using the basis representation (17) of $\boldsymbol{u}$, we can represent the elastic operator $\mathcal{A}=\mu \Delta \boldsymbol{u}+(\lambda+\mu) \nabla(\nabla \cdot \boldsymbol{u})$ by the following parameterizations

$$
\begin{array}{r}
\Delta \boldsymbol{u}=\sum_{i=1}^{s} \sum_{I \in \mathcal{I}} \boldsymbol{c}_{I} \frac{\partial^{2}}{\partial x_{i}^{2}} B_{I}=\sum_{I \in \mathcal{I}} \boldsymbol{c}_{I} \sum_{i=1}^{s} \frac{\partial^{2}}{\partial x_{i}^{2}} B_{I} \\
\nabla(\nabla \cdot \boldsymbol{u})=\sum_{i, j=1}^{s} \sum_{I \in \mathcal{I}} \boldsymbol{e}_{i} c_{I j} \frac{\partial^{2}}{\partial x_{i} \partial x_{j}} B_{I}
\end{array}
$$

For the inverse compositional optimization the regularization term must be formulated as $\mathcal{S}[\boldsymbol{u}]=\sum_{i} F_{i}^{2}(\boldsymbol{c})$. We parameterize the terms $\nabla \cdot \boldsymbol{u}$ and $\frac{\partial \boldsymbol{u}_{i}}{\partial \boldsymbol{x}_{j}}+\frac{\partial \boldsymbol{u}_{j}}{\partial \boldsymbol{x}_{i}}$ by

$$
\begin{array}{r}
\nabla \cdot \boldsymbol{u}=\sum_{I \in \mathcal{I}, j \in\{1, \ldots, s\}} c_{I j} \frac{\partial}{\partial x_{j}} B_{I} \\
{\left[\frac{\partial \boldsymbol{u}_{i}}{\partial \boldsymbol{x}_{j}}+\frac{\partial \boldsymbol{u}_{j}}{\partial \boldsymbol{x}_{i}}\right]=\sum_{I \in \mathcal{I}} \boldsymbol{c}_{I i} \frac{\partial}{\partial x_{j}} B_{I}+\boldsymbol{c}_{I j} \frac{\partial}{\partial x_{i}} B_{I} .}
\end{array}
$$

It is clear that both terms are linear in $c$, which yields the representation of $F_{i}^{2}$, when the integral is discretized.

\section{References}

[1] S. Baker and I. Matthews. Lucas-kanade 20 years on A unifying framework. International Journal of Computer Vision, 56(3):221-255, 2004.

[2] S. Baker, D. Scharstein, J. Lewis, S. Roth, M. J. Black, and R. Szeliski. A database and evaluation methodology for optical flow. In ICCV, 2007.
[3] T. Cootes, C. Twining, V. Petrovic, R. Schestowitz, and C. J. Taylor. Groupwise construction of appearance models using piece-wise affine deformations. In Proceedings of British Machine Vision Conference (BMVC), volume 2, pages 879_ 888, 2005.

[4] W. Dahmen, C. Micchelli, and H.-P. Seidel. Blossoming begets B-splines built better by B-patches. In Math. Comput., volume 59, pages 97-115, 1992.

[5] D. Forsey and R. Bartels. Hierarchical B-spline refinement. ACM Tran. Comp. Graph., pages 205-212, 1988.

[6] M. Franssen, R. C. Veltkamp, and W. Wesselink. Efficient evaluation of triangular B-spline surfaces. In Comp. Aided Geometric Design, volume 17, pages 863-877, 2000.

[7] M. Neamtu. What is the natural generalization of univariate splines to higher dimensions? In Math. Methods for Curves and Surfaces: Oslo 2000, pages 355-392, 2000.

[8] M. Neamtu. Delaunay configurations and multivariate splines: A generalization of a result of $\mathrm{n}$. b. delaunay. Trans. of the American Math. Society, pages 2993-3004, 2007.

[9] L. Pantoni, A. M. Basile, G. Pracucci, K. Asplund, J. Bogousslavsky, H. Chabriat, T. Erkinjuntti, F. Fazekas, J. M. Ferro, M. Hennerici, J. O’brien, P. Scheltens, M. C. Visser, L. O. Wahlund, G. Waldemar, A. Wallin, and D. Inzitari. Impact of age-related cerebral white matter changes on the transition to disability - the LADIS study: Rationale, design and methodology. Neuroepidemiology, 24(1-2):51-62, 2005.

[10] R. R. R. Chandrashekara, R. Mohiaddin and D. Rueckert. Nonrigid image registration with subdivision lattices: Application to cardiac $\mathrm{mr}$ image analysis. In proc. of MICCAI, page in press, 2007.

[11] D. Rueckert, L. I. Sonoda, C. Hayes, D. L. G. Hill, M. O. Leach, and D. J. Hawkes. Nonrigid registration using freeform deformations: application to breast MR images. IEEE Trans. on Medical Imaging, 18(8):712-21, 1999.

[12] J. A. Schnabel, D. Rueekert, M. Quist, J. M. Blackall, A. D. Castellano-Smith, T. Hartkens, G. P. Penney, W. A. Hall, H. Liu, C. L. Truwit, F. A. Gerritsen, D. L. G. Hill, and D. J. Hawkes. A generic framework for non-rigid registration based on non-uniform multi-level free-form deformations. Proc. of MICCAI, 2208:573-81, 2001.

[13] T. W. Sederberg and S. R. Parry. Free-form deformation of solid geometric models. Computer Graphics (Proc. SIGGRAPH '86), pages 151-160, 1986.

[14] R. I. Silveira and M. van Kreveld. Optimal higher order delaunay triangulations of polygons. Tech. Report, 2007.

[15] K. Wang, Y. He, and H. Qin. Incorporating rigid structures in non-rigid registration using triangular B-splines. In Variational, Geometric, and Level Set Methods in Computer Vision, volume 59, pages 235-246, 2005.

[16] Z. Xie and G. Farin. Image registration using hierarchical B-splines. IEEE Transactions on Volume Visualization and Computer Graphics, 10(1):85-94, 2004. 